\title{
Self-organized graphene crystal patterns
}

\author{
Bin $\mathrm{Wu}^{1,3}$, Dechao Geng ${ }^{1,3}$, Zhiping $\mathrm{Xu}^{2}$, Yunlong Guo ${ }^{1}$, Liping Huang ${ }^{1}$, Yunzhou Xue ${ }^{1}$, Jianyi Chen ${ }^{1}$, \\ Gui $\mathrm{Yu}^{1}$ and Yunqi Liu ${ }^{1}$
}

The phenomenon of ordered pattern formation is universal in nature but involves complex non-equilibrium processes that are highly important for both fundamental research and applied materials systems. Among countless pattern systems, a snowflake is possibly the most fascinating example offered by nature. Here, we report that single-layered and single-crystalline graphene flakes (GFs) with highly regular and hexagonal symmetric patterns can be grown on a liquid copper surface using a $\mathrm{CH}_{4}$ chemical vapor deposition (CVD) method. The different morphologies of these GFs can be precisely tailored by varying the composition of the inert gas $/ \mathrm{H}_{2}$ carrier gas mixture used to produce the GFs, and the GF edges can be continuously tuned over the full spectrum from negative to zero to positive curvature in a controllable way. The family of GF crystal patterns is remarkably analogous to that of snowflakes, representing an ideal two-dimensional (2D) growth system. Pattern formations from compact to dendritic GFs can be explained by the continuous modulation of the competition between adatom diffusion along island edges or corners and surface diffusion processes.

NPG Asia Materials (2013) 5, e36; doi:10.1038/am.2012.68; published online 1 February 2013

Keywords: graphene pattern; crystal growth; snowflakes; chemical vapor deposition

\section{INTRODUCTION}

Self-organization into ordered patterns is ubiquitous in nature and involves a transition from disordered building blocks to ordered/ crystalline assemblies. In addition to the most thermodynamically stable structure, a system of many particles can adopt various metastable structures as aggregate forms in non-equilibrium processes, depending on various thermodynamic variables, and the transport of materials and energy in the system. The ability to control pattern formation and a better understanding of the process are of technological and scientific importance. For example, controlling the process of pattern formation has been extensively studied in several model systems, including snowflake formation, ${ }^{1-3}$ metal aggregation on a substrate, ${ }^{4,5}$ and colloidal nanocrystal growth. ${ }^{6,7}$ However, owing to the many atomic-level complex processes involved, the formation of self-organized patterns at nanometer/micrometer length scales is very sensitive to various conditions, thus posing a great challenge for controllably tailoring the structures of patterned materials. Of the countless pattern systems of materials, a snowflake-which can exhibit a large number of various beautiful shapes with high, sixfold symmetry depending on the temperature and supersaturationis likely the most fascinating example formed by a non-equilibrium process as it offers conceptual simplicity and allows theoretical studies to probe the kinetic mechanisms of atomic growth. The controlled formation of other well-defined snowflake-like systems of materials, however, has met with very limited success. ${ }^{8}$
As an important two-dimensional (2D) material, graphene shows unique properties that are controlled by its layer number, degree of lattice order, size, shape and edge structure. ${ }^{9-12}$ Controlling these parameters is not only highly important for obtaining the desired characteristics but can also have a profound impact on our understanding of non-equilibrium growth processes as graphene represents the simplest 2D model system. Given that small graphene flakes (GFs) can be grown on a Cu surface via a surface nucleation growth mechanism, ${ }^{13,14}$ a chemical vapor deposition (CVD) approach thus provides a platform to manipulate the shape of these GFs and tests the validity of the classical theory of nucleation and growth. Previous work has shown that GFs exhibiting a variety of shapes, including hexagons, ${ }^{15-20}$ four-lobed grains ${ }^{21,22}$ and six-sided polygons, ${ }^{20}$ can be formed on solid $\mathrm{Cu}$ surfaces under appropriate conditions.

Precise control over GF shapes fabricated using a CVD method relies on an understanding of growth kinetics. At the atomic level, $\mathrm{CH}_{4}$ molecules are first adsorbed on a $\mathrm{Cu}$ surface and then dissociated into free $\mathrm{C}$ atoms during the CVD growth process. The adatoms (adsorbed $\mathrm{C}$ atoms) can migrate on the surface and aggregate into small critical nuclei that are further enlarged over the 2D space. The grain expansion process is mainly affected by the rates of adatom diffusion on the surface, along steps, and around island corners, as well as the deposition flux of $\mathrm{C}$ atoms, and the interplay between these factors is responsible for the final morphology of GFs. ${ }^{3,5}$ In

\footnotetext{
${ }^{1}$ Beijing National Laboratory for Molecular Sciences, Key Laboratory of Organic Solids, Institute of Chemistry, Chinese Academy of Sciences, Beijing, People's Republic of China and ${ }^{2}$ Department of Engineering Mechanics and Center for Nano and Micro Mechanics, Tsinghua University, Beijing, People's Republic of China

3These authors contributed equally to this work.

Correspondence: Dr Y Liu, Beijing National Laboratory for Molecular Sciences, Key Laboratory of Organic Solids, Institute of Chemistry, Chinese Academy of Sciences, Beijing 100190, People's Republic of China.

E-mail: liuyq@iccas.ac.cn
}

Received 26 June 2012; revised 11 October 2012; accepted 4 November 2012 
general, the rates of these processes can be manipulated by varying the temperature, the flux of the $\mathrm{CH}_{4}$ precursor gas and carrier gas and the substrate. However, it is generally difficult to separately manipulate the rates of microscopic steps and then link the effect of varying these parameters directly with these rates, leading to the ineffective manipulation of the overall process. Here, we demonstrate a general shape engineering approach that allows for the controlled growth of GFs with various well-defined shapes on a liquid $\mathrm{Cu}$ surface using a $\mathrm{CH}_{4}$-CVD method. The collection of GF shapes closely resembles the family of snowflake shapes and constitutes a well-defined and simple 2D model system for studying other materials. The GF morphologies can be directly modulated by changing the flow rate ratio between the inert gas (Ar or $\mathrm{He}$ ) and $\mathrm{H}_{2}$, following a universal rule for shape evolution.

\section{MATERIALS AND METHODS}

\section{Materials}

$\mathrm{Cu}$ foil (99.8\% purity, $25 \mu \mathrm{m}$ thick), brass foil (alloy 260, $0.13 \mathrm{~mm}$ thick) and W foil ( $99.95 \%$ purity, $50 \mu \mathrm{m}$ thick) were obtained from Alfa Aesar, Tianjin, China.

\section{CVD graphene synthesis and transfer}

$\mathrm{Cu}$ foils or brass foils on $\mathrm{W}$ substrates were loaded into a one inch quartz CVD tube. The tube was then heated in pure $\mathrm{H}_{2}$ gas in a furnace (Lindberg/Blue M, TF55035A, Thermo Fisher Scientific Inc., Asheville, NC, USA) to $1080^{\circ} \mathrm{C}$ (the melting point of $\mathrm{Cu}$ ). The samples were then annealed for $30 \mathrm{~min}$. At the beginning of growth, the $\mathrm{Ar}$ (or $\mathrm{He}$ gas) and $\mathrm{H}_{2}$ flow rates were adjusted to the desired values, and $\mathrm{CH}_{4}$ was then introduced to the chamber at the required rate for a certain time. Finally, the flow of $\mathrm{CH}_{4}$ was turned off, and the system was cooled to room temperature at a cooling rate of $\sim 25^{\circ} \mathrm{C} \mathrm{min}{ }^{-1}$. Different temperatures such as 1050,1065 and $1080^{\circ} \mathrm{C}$ were employed to study their role in affecting GF shapes. In each case, the temperature was changed by switching off the furnace after the annealing process, dropping the temperature from $1080^{\circ} \mathrm{C}$ to the desired value in $\sim 2-4 \mathrm{~min}$. Then, the furnace was turned on until the desired temperature was obtained. The growth process was then induced using a procedure similar to that described above. The GFs grown on flat $\mathrm{Cu} / \mathrm{W}$ surfaces were transferred to $300 \mathrm{~nm} \mathrm{SiO}_{2} / \mathrm{Si}$ substrates and TEM (transmission electron microscope) grids by a poly(methyl methacrylate)assisted method. The poly(methyl methacrylate)-supported films were removed with acetone.

\section{Characterization of GFs}

The samples were characterized by scanning electron microscopy (SEM) (Hitachi S-4800, 1 kV, Hitachi, Ltd., Tokyo, Japan), optical microscopy, AFM (Veeco Nanoman VS, Veeco Instruments Inc., New York, NY, USA) in tapping mode, Raman spectroscopy (Renishaw inVia Plus, with laser excitation at $514 \mathrm{~nm}$ and a spot size of $1-2 \mu \mathrm{m}$, Renishaw plc, Gloucestershire, UK) and TEM (Tecnai G2 F20 U-TWIN, operated at 200 kV, FEI, Hillsboro, OR, USA).

\section{Kinetic Monte Carlo simulation of GF growth and electrical measurements of GFs}

The detailed simulation procedure is described in the supporting information. The electrical properties of the GFs transferred onto $300 \mathrm{~nm} \mathrm{SiO} / 2 \mathrm{Si}$ substrates were measured. Graphene back-gated field-effect transistor (FET) devices were fabricated using our previously reported method. ${ }^{23}$ Briefly, $2-5 \mu \mathrm{m}$ wide organic nanowires were deposited on individual GFs; then, a $30-40 \mathrm{~nm}$ gold film was evaporated on the sample. Finally, the nanowires were removed by a micromanipulator, and the desired electrodes were fabricated by mechanically scratching the gold film to produce isolated FET devices. The tests, including the measurement of two-terminal $I-V$ curves and the back-gated FET properties of GFs, were conducted with a Keithley 4200 analyzer at room temperature in air, and the $2 \mathrm{D}$ resistivity and saturation current density of the GFs were calculated from the data. The mobility of hole carriers was extracted from the equation: $\mu_{d e v}=\frac{L}{V_{D} C_{o x} W} \cdot \frac{d I_{d}}{d V_{g}}$, where $L$ and $W$ are the device channel length and width, respectively, $V_{D}$ is the voltage between the source and drain electrodes, and $C_{o x}$ is the gate capacitance per unit area. ${ }^{23}$

\section{RESULTS AND DISCUSSION}

Our primary $\mathrm{CH}_{4}-\mathrm{CVD}$ approach involves the growth of GFs on a liquid $\mathrm{Cu}$ surface in a mixture of $\mathrm{Ar}$ and $\mathrm{H}_{2}$ gas at ambient pressure, as schematically shown in Figure 1a. We recently demonstrated that using a liquid $\mathrm{Cu}$ phase catalyst is an effective way to achieve the homogeneous nucleation and growth of single-crystalline hexagonal GFs in a pure $\mathrm{H}_{2}$ gas environment. ${ }^{23}$ It is important to recognize that GFs grown on liquid $\mathrm{Cu}$ surfaces predominantly have highly symmetric shapes. In contrast, when GF structures are grown on a solid $\mathrm{Cu}$ surface, the intrinsic regular hexagonal symmetry is usually distorted by the anisotropic surface, resulting in deviations from a regular hexagonal shape. ${ }^{17}$ Our subsequent experiments, reported here, showed that the introduction of $\mathrm{Ar}$ into the pure $\mathrm{H}_{2}$ gas atmosphere resulted in a dramatic change in GF shape. Figure 1 shows a typical result, in which dendritic GFs were grown on a liquid $\mathrm{Cu} / \mathrm{W}$ surface at $1080^{\circ} \mathrm{C}$ using $0.5 \mathrm{sccm}$ (standard cubic centimeters per minute) $\mathrm{CH}_{4}, 10 \mathrm{sccm} \mathrm{H}_{2}$ and $800 \mathrm{sccm}$ Ar for $\sim 5 \mathrm{~min}$. These dendritic GFs were well dispersed on the $\mathrm{Cu}$ surface and formed in high yield (Figures $1 \mathrm{~b}$ and $\mathrm{c}$ ). The structures show six-fold symmetry (Figure 1d) and are highly reproducible. Both SEM and optical images show uniform contrast of the GFs with respect to the $\mathrm{Cu}$ surface, indicating the single-layer nature of the as-grown GFs. A Raman spectrum of a typical GF transferred onto a $300 \mathrm{~nm} \mathrm{SiO} / 2 \mathrm{Si}$ substrate (Figure 1e) shows single-layer features, consistent with those reported for hexagonal GFs grown on solid and liquid $\mathrm{Cu}$ surfaces. ${ }^{17,23}$

The manipulation of the $\mathrm{Ar}: \mathrm{H}_{2}$ flow rate ratio results in the evolution of GF shapes in a definite manner. Figures $2 \mathrm{a}-\mathrm{k}$ show a series of SEM images of typical GFs grown on liquid $\mathrm{Cu}$ surfaces with the different flow rate ratios of Ar: $\mathrm{H}_{2}$ shown in Table S1. Using higher ratios of $\mathrm{Ar}: \mathrm{H}_{2}$, GFs with a symmetric dendritic structure are formed (Figures 2a-d). As the Ar: $\mathrm{H}_{2}$ ratio is decreased, more-compact structures appear (Figures 2e-h), and eventually, regular hexagonal GFs with straight edges are obtained (Figures $2 \mathrm{i}$ and $\mathrm{j}$ ). Using pure $\mathrm{H}_{2}$ leads to a slight deviation from perfect hexagonal structures, producing edges with positive curvature (Figure $2 \mathrm{k}$ ). Each GF has six-fold hexagonal symmetry, and SEM images of large areas (Supplementary Figure S1) show that the geometry of the GFs formed under a specific set of conditions is highly uniform and reproducible. Remarkably, a clear trend in GF shape evolution can be recognized from Figures $2 \mathrm{k}$ to $2 \mathrm{a}$; that is, the edges of the regular hexagon progressively curve toward the center of the hexagon as the proportion of Ar is increased. The entire set of GF morphologies is an excellent analog to the family of snowflake shapes. Moreover, we also found that the edges of the hexagonal GFs adopted a positive curvature upon increasing the $\mathrm{CH}_{4}$ flow rate in a pure $\mathrm{H}_{2}$ environment, eventually forming circular GFs as shown in Figure 21 when using $22 \mathrm{sccm} \mathrm{CH}_{4}$ rather than $5 \mathrm{sccm}$, as in the case of the GF shown in Figure $2 \mathrm{k}$. These results demonstrate that GF edges can be tuned over the full spectrum from negative to zero to positive curvature in a controllable way. This provides an ideal system for the study of non-equilibrium growth processes and graphene edge/property correlations.

The GFs were characterized by atomic force microscopy (Supplementary Figure S2), Raman spectroscopy and transmission electron microscopy. The Raman spectra of GFs with different morphologies were all similar to the spectrum shown in Figure 1e, confirming the presence of single-layer graphene in each case. The formation of monolayer GFs was also confirmed by a high-resolution 

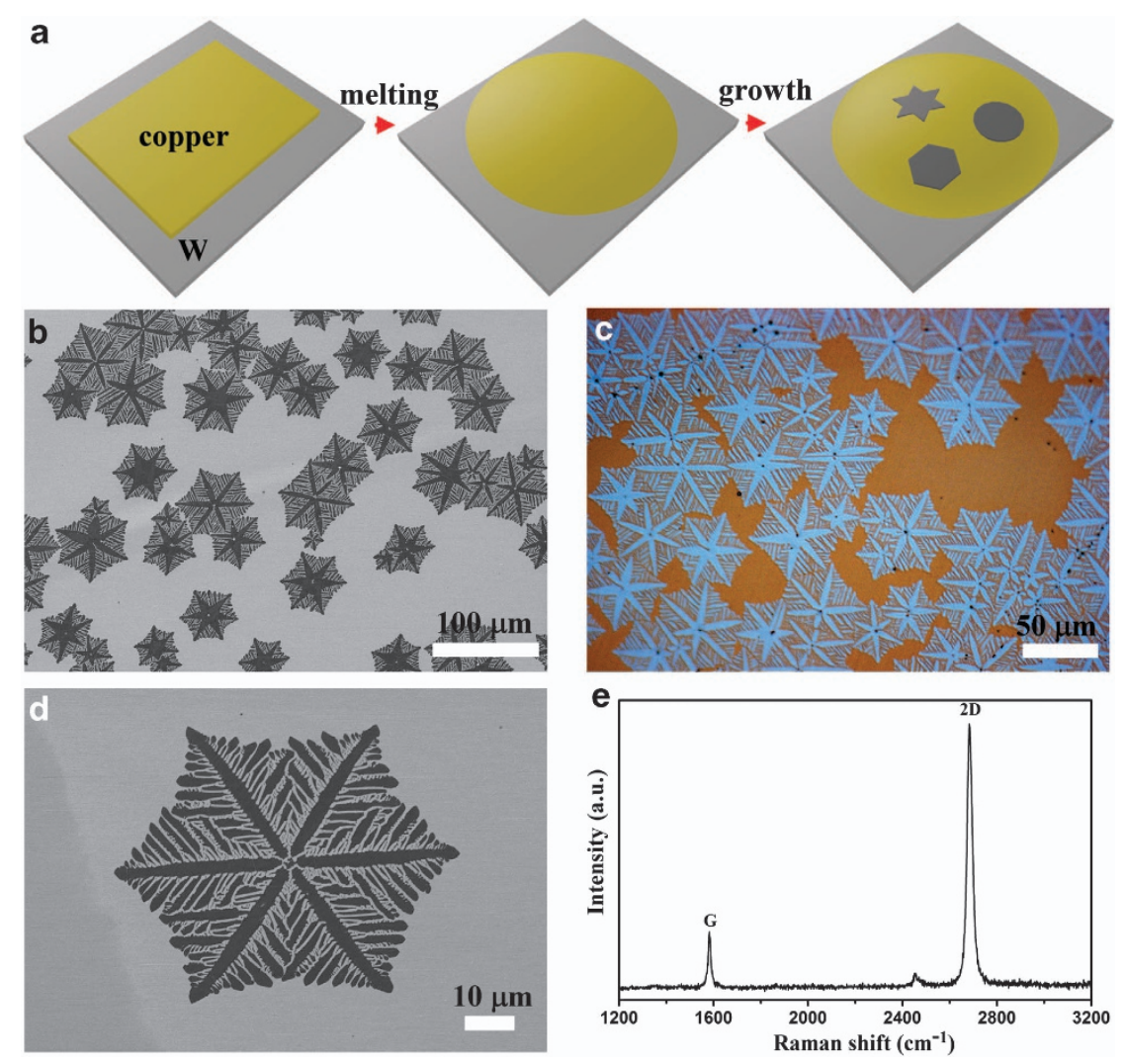

Figure 1 The growth of dendritic GFs on a liquid Cu surface. (a) A schematic illustration showing the process of GF growth using the $\mathrm{CH}_{4}$-CVD method. Left image: solid $\mathrm{Cu}$ pieces on a W substrate. As the temperature is increased above the melting point of $\mathrm{Cu}$, solid $\mathrm{Cu}$ turns into a liquid phase that forms a flat surface owing to the good wetting behavior between $\mathrm{Cu}$ and the substrate (middle image). Finally, the GFs are grown on the liquid $\mathrm{Cu}$ surface under different conditions. Typical SEM (b) and optical (c) images showing well-dispersed dendritic GFs produced on a liquid $\mathrm{Cu}_{\text {surface }}$ using $0.5 \mathrm{sccm} \mathrm{CH}_{4}$, $10 \mathrm{sccm} \mathrm{H}_{2}$, and $800 \mathrm{sccm} \mathrm{Ar}$ at $1080^{\circ} \mathrm{C}$ for $\sim 5 \mathrm{~min}$. (d) A magnified SEM image of an individual GF. (e) A Raman spectrum of a GF transferred onto a $300 \mathrm{~nm} \mathrm{SiO} /$ Si substrate, confirming its single-layer characteristics.

TEM image of a GF edge, shown in the inset of Supplementary Figure S3a. The results of selected-area electron-diffraction measurements on different-shaped GFs were explored under a TEM, and the results for typical hexagonal (Figure 2k), flower-like (Figure 2f) and dendritic GFs (Figure $2 c$ ) are shown in Figures $2 \mathrm{~m}-\mathrm{q}$, Supplementary Figure S3 and Figures $2 \mathrm{r}-\mathrm{v}$, respectively. The sets of six-fold symmetric selectedarea electron-diffraction patterns at different locations of the GFs adopt essentially the same orientation, confirming the single-crystalline nature of the materials. Because the GF shape changes in a continuous manner, it is to be expected that all the other GFs are also single crystalline (neglecting the branched parts).

In addition, the use of $\mathrm{Cu}$ alloy (brass, $30 \% \mathrm{Zn}$ and $70 \% \mathrm{Cu}$ ) as a liquid catalyst also resulted in a similar manipulation of GF morphologies using the approach described in this paper, as shown in Figure 3, Supplementary Table S2 and Supplemantary Figure S4, reflecting the possibility of fine tuning GF morphology. For example, when the $\mathrm{Ar}: \mathrm{H}_{2}$ ratio was low, the GFs assumed a compact hexagonal shape (Figure 3a). As the Ar: $\mathrm{H}_{2}$ ratio increased, the vertices of the hexagonal GF crystals were shaped (Figures 3b-e), producing increasingly more concave polyhedral structures. Further increasing this ratio led to the formation of flower-like GFs (Figure 3f), similar to the case in which pure $\mathrm{Cu}$ was used.

Experiments were performed to gain insight into the GF growth mechanism by varying the following parameters: temperature, the use of inert $\mathrm{He}$ gas instead of $\mathrm{Ar}$ and the $\mathrm{CH}_{4}$ flux. Within the temperature range from $1050^{\circ} \mathrm{C}$ to $1080^{\circ} \mathrm{C}$ (see the description in Methods), we observed that the GF shapes evolved in exactly the same way upon varying the Ar to $\mathrm{H}_{2}$ flow ratio. The use of $\mathrm{He}$ gas also leads to the production of the same spectrum of GF shapes. These results rule out any critical roles that either temperature or the 'chemistry' of Ar has in shaping GF geometry or edge structures. Finally, the conditions used to prepare the GF shown in Figure $2 \mathrm{~g}$ $\left(800 \mathrm{sccm} \mathrm{Ar}, 50 \mathrm{sccm} \mathrm{H} \mathrm{H}_{2}\right.$ ) were varied by increasing the $\mathrm{CH}_{4}$ flow rate from $0.5 \mathrm{sccm}$ (as in Figure $2 \mathrm{~g}$ ) to $2 \mathrm{sccm}$. As shown in Supplementary Figure S5, GFs with similar morphology were obtained, but there was a slight increase in the negative curvature of the edges with increasing $\mathrm{CH}_{4}$ deposition flux.

The variation in the atomic-level mechanism of GF pattern formation as a function of the composition of $\mathrm{Ar}$ and $\mathrm{H}_{2}$ in the gas can be understood in a qualitative manner. As schematically shown in Figure 4a, the competition between adatom diffusion along island edges (process 1 indicated in Figure 4a) and adatom surface diffusion (process 2 indicated in Figure 4a) determines the final kinetically controlled morphologies of GFs. A compact island is formed when an adatom relaxes sufficiently to find an energetically more favorable location along island edges or corners before additional adatoms migrate by surface diffusion to join it. In the absence of such relaxation, growth leads to dendritic structures. ${ }^{3,5}$ An analysis of our results suggests that kinetic control over the growth of GFs is owing to the effect of the composition of the inert gas $/ \mathrm{H}_{2}$ carrier gas 

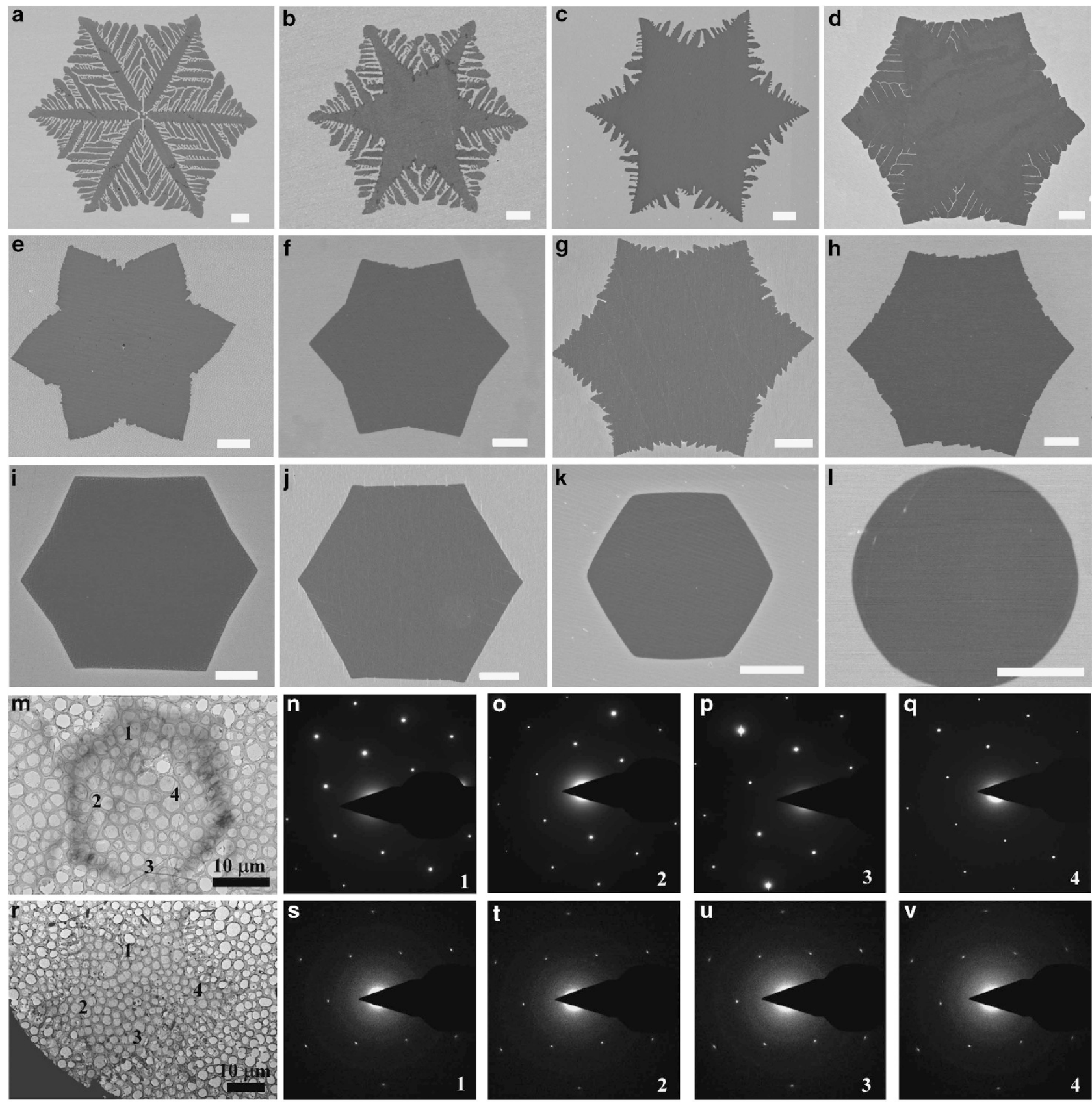

Figure 2 SEM and TEM images and selected area electron diffraction patterns of a series of GFs grown on a liquid Cu surface with different shapes formed by varying the $\mathrm{Ar}: \mathrm{H}_{2}$ ratio. (a-I) Typical SEM images of separated GFs under different conditions as summarized in Supplementary Table S1 (in the supporting information). All scale bars are $5 \mu \mathrm{m}$. TEM image (m) of one hexagonal GF similar to that shown in Figure 2 (k), illustrating the apparent shape and the selected area electron diffraction (SAED) patterns $(\mathbf{n}-\mathbf{q})$ at four different locations labeled 1, 2, 3 and 4 in the TEM image. ( $\mathbf{r}-\mathbf{v})$ TEM image of one GF similar to that shown in Figure 2 (c), together with SAED patterns obtained at different locations.

mixture on adatom surface diffusion. This control is realized by tuning the ratio of the density of adsorbed $\mathrm{CH}_{4}$ molecules to that of free $\mathrm{C}$ atoms on the liquid $\mathrm{Cu}$ surface. For example, the partial pressure of $\mathrm{H}_{2}$ in the mixed gas is capable of modulating the percentage of $\mathrm{CH}_{4}$ molecules adsorbed on the surface being converted to free $\mathrm{C}$ atoms by affecting the chemical equilibrium of $\mathrm{CH}_{4}$ dissociation, that is, lower partial pressures of $\mathrm{H}_{2}$ (higher partial pressures of $\mathrm{Ar}$ ) favor the dissociation of $\mathrm{CH}_{4}$, leaving fewer undissociated $\mathrm{CH}_{4}$ molecules on the surface. Thus, the presence of $\mathrm{CH}_{4}$ results in an energy barrier to $\mathrm{C}$ atom surface diffusion, which hinders the mobility of $\mathrm{C}$ atoms. ${ }^{5}$ With a reduced energy barrier, the use of a higher Ar flux thus leads to the more rapid surface diffusion of $\mathrm{C}$ atoms toward GF islands (process 2 in Figure 4a), and eventually, adatom surface diffusion is so rapid that the relaxation process of $\mathrm{C}$ atoms along island edges (process 1 in Figure 4a) becomes insufficient, leading to the formation of dendritic GF patterns.

Based on the proposed mechanism, GF branching was studied by kinetic Monte Carlo simulations (see the detailed description of the simulation methods in the supporting information). Figures $4 \mathrm{~b}$ and $\mathrm{c}$ show the simulation results for two cases, in which the $\mathrm{C}$ adatom 

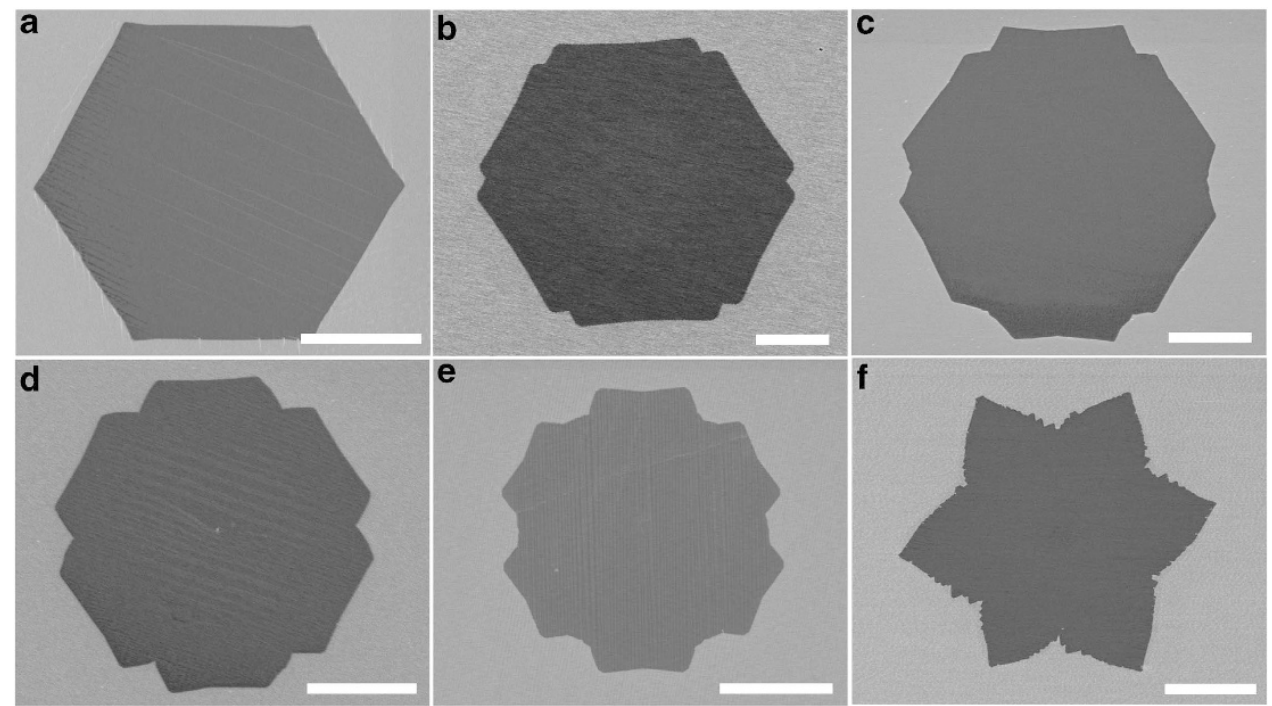

Figure 3 Variously shaped GFs formed on a liquid brass surface under different growth conditions. (a-f) Typical SEM images of individual GFs prepared under the conditions summarized in Supplementary Table S2 (in the supporting information). All scale bars are $10 \mu \mathrm{m}$.
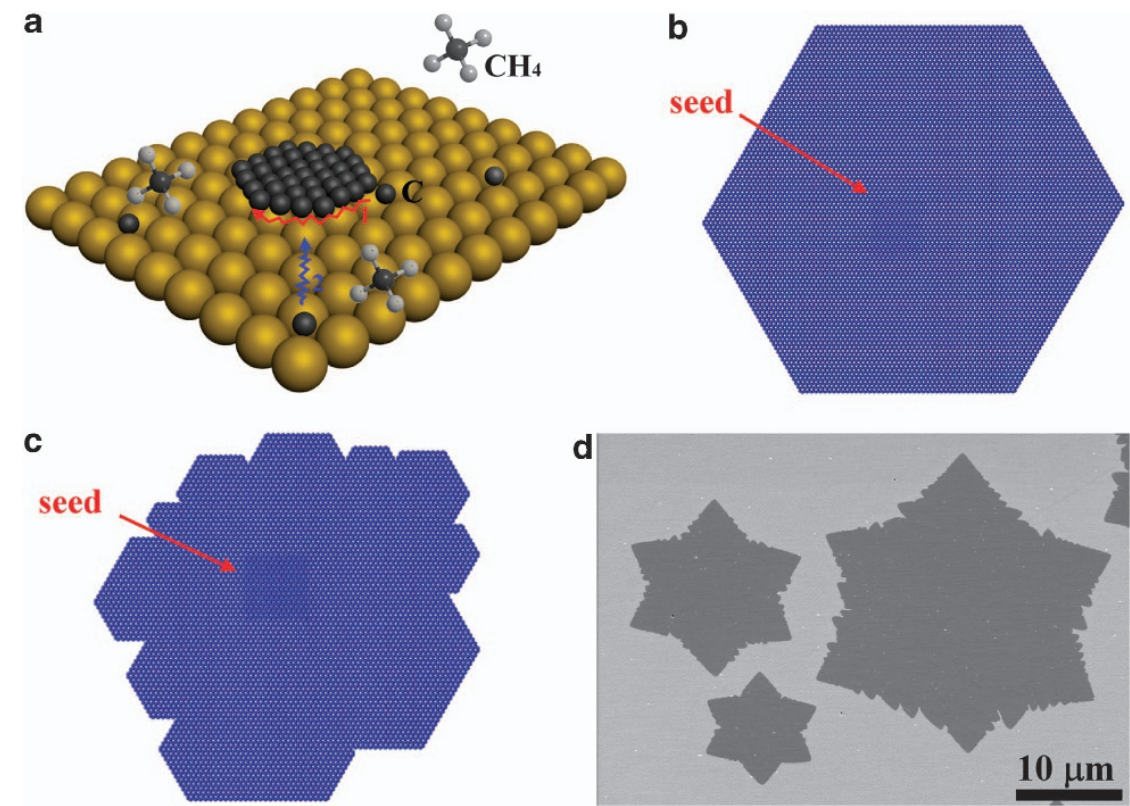

Figure 4 Kinetic growth mechanism of GFs. (a) A schematic picture showing the presence of adsorbed $\mathrm{CH}_{4}$ molecules, free $\mathrm{C}$ atoms and a graphene island on the Cu surface during the CVD process. Two processes of $\mathrm{C}$ atom diffusion are indicated by $\mathbf{1}$ and $\mathbf{2}$, which refer to adatom diffusion along edges/corners and on the surface, respectively. (b) Hexagonal GF observed in the kinetic Monte Carlo simulation with a low adatom diffusion rate on the surface, representing growth conditions featuring a low Ar: $\mathrm{H}_{2}$ mixed gas ratio. (c) Branched GF structure formed in kinetic Monte Carlo simulation with a high adatom diffusion rate on the surface, representing growth conditions featuring a high $\mathrm{Ar}: \mathrm{H}_{2}$ mixed gas ratio. (d) A typical SEM image of different-sized GFs grown under the same conditions $(0.5 \mathrm{sccm} \mathrm{CH} / 35 \mathrm{sccm} \mathrm{H} / 200 \mathrm{sccm}$ Ar), clearly showing that GF edges become rougher with increasing GF size.

diffusion rate on a structureless substrate is modulated to be lower or higher than that at graphene edges, reflecting a lower or higher $\mathrm{Ar}: \mathrm{H}_{2}$ ratio during the growth process, respectively. The simulation (Figures $4 \mathrm{~b}$ and c) clearly shows that a hexagonal GF is formed when the Ar: $\mathrm{H}_{2}$ ratio is low, and at a higher $\mathrm{Ar}: \mathrm{H}_{2}$ ratio, the GFs grow into rather branched structures.

The proposed mechanism can also explain the finding that shaped GFs grown on liquid brass (Figure 3f) are much less dendritic than those grown on liquid $\mathrm{Cu}$ (Figure 2a) using the same $\mathrm{Ar}: \mathrm{H}_{2}$ flow rate ratio. $\mathrm{Zn}$ atoms on liquid brass surface behave as impurity sites (similar to adsorbed $\mathrm{CH}_{4}$ molecules on a surface) that reduce the adatom surface diffusion mobility. In addition, the dynamic evolution of growing GFs could be detected by comparing different-sized GFs of the same sample. The edges of larger GFs nucleated in the early stages of growth are more rough than those of smaller GFs nucleated in the later stages of the growth process, as clearly shown in Figure 4d and Supplementary Figure S6. This edge roughening of GFs over time is similar to the case of snowflake formation and is owing to higher 
growth rates occur at the intrusions along the edges of the GFs in diffusion-limited growth as growth proceeds. ${ }^{1}$

The evolution of GF patterns as a function of $\mathrm{CH}_{4}$ flux (Supplementary Figure S5) is also consistent with the above mechanism. As the $\mathrm{CH}_{4}$ flux increases, the ratio of the density of adsorbed $\mathrm{C}$ atoms to that of $\mathrm{CH}_{4}$ molecules remains essentially the same when using the same composition of $\mathrm{Ar}$ and $\mathrm{H}_{2}$ gas. However, the increased overall density of $\mathrm{C}$ atoms on the surface reduces the average time for adatom diffusion to GF islands, resulting in GF edges with a morenegative curvature. Note that the degree of modulation of GF shapes with varying $\mathrm{CH}_{4}$ flux is much weaker than the effect of varying the composition of the $\mathrm{Ar} / \mathrm{H}_{2}$ mixture, indicating that the latter is the main driving force for GF-shape engineering.

Electrical measurements were also performed to compare the electrical properties of GFs grown in mixed $\mathrm{Ar}$ and $\mathrm{H}_{2}$ with those of our previously reported hexagonal GFs grown in pure $\mathrm{H}_{2}{ }^{23}$ using the same device fabrication process. Figures $5 \mathrm{a}$ and $\mathrm{b}$ show typical SEM and optical images of a single-layer GF (corresponding to the sample in Figure $2 \mathrm{~h}$ ) device, respectively. A typical current-voltage $(I-V)$ curve measured for a device is shown in Figure 5c, showing a linear relationship and low resistance $(\sim 60 \Omega)$. Figure $5 \mathrm{~d}$ shows a plot of 2D GF resistivity (defined as $R \times W / L$, where $W$ is the width of the GF, $L$ is the channel length of the device and $R$ is the resistance) as a function of GF width, giving an average value of $742 \pm 95 \Omega$, which is similar to the value of $609 \pm 200 \Omega^{23}$ for hexagonal GFs. Large saturation current densities (defined as saturation current divided by graphene device width) were also calculated from the $I-V$ curves of these two-terminal devices. Figure $5 \mathrm{e}$ shows a typical $I-V$ curve of a GF device exhibiting current saturation. The average value of the saturation current for the GFs was estimated to be $0.88 \pm 0.32 \mathrm{mA \mu m}^{-1}$, with the highest value reaching up to $1.7 \mathrm{mA \mu m}^{-1}$ (which is $\sim 3.9$ times higher than the value of $0.44 \mathrm{~mA}^{-1} \mathrm{~m}^{-1}$ reported for CVD-grown graphene ${ }^{24}$ shown in Figure 5f). Moreover, the electronic quality of transferred GFs with different edge curvatures was also evaluated using these back-gated FET devices under ambient conditions. Figure $5 \mathrm{~g}$ shows a typical SEM image of a GF device, in which the edge of a GF with negative curvature can be clearly identified. The representative transfer curve is shown in Figure $5 \mathrm{~h}$. For most devices, the Dirac point was not clearly observed in the range of applied gate voltages, possibly because of oxygen adsorption or doping effects from the transfer process. Figure $5 \mathrm{i}$ shows the extracted hole mobility values for 30 devices. These scattered values essentially fall into the range of $800-$ $2000 \mathrm{~cm}^{2} \mathrm{~V}^{-1} \mathrm{~s}^{-1}$ for GFs with different edge curvatures. Both twoand three-terminal measurements of GF devices demonstrated that the quality of GFs grown in mixed $\mathrm{Ar}$ and $\mathrm{H}_{2}$ is compatible with that of hexagonal GFs grown in pure $\mathrm{H}_{2}$.

In conclusion, this study provides a well-behaved $2 \mathrm{D}$ crystal growth system mimicking snowflakes, opening up rich opportunities for the engineering of graphene patterns and for the study of graphene
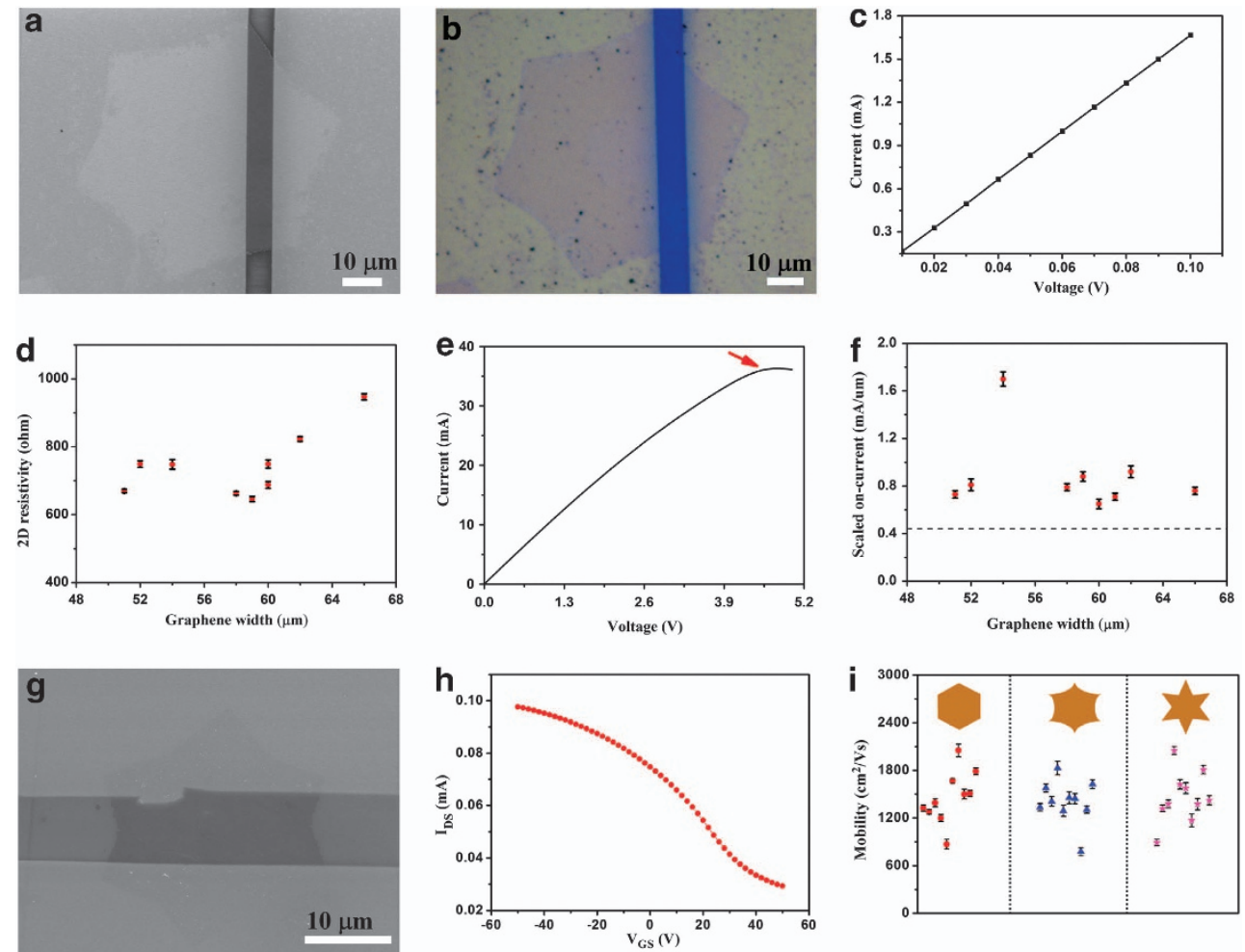

Figure 5 Electrical characterization of GFs. (a) SEM image of one typical two-terminal device based on an individual GF contacted by 40 nm left and right gold electrodes. (b) The corresponding optical image of the same GF device. (c) I-V curve of a device with a resistance of $\sim 60 \Omega$. (d) A plot comparing the $2 \mathrm{D}$ resistivity of GFs as a function of GF width among many devices. (e) A typical $I-V$ curve of a GF device showing current saturation before the GF was broken down. The arrow indicates the turning point of the current and is used to calculate the saturation current density. (f) $A$ plot of saturation current density (scaled on-current) versus GF width measured for many graphene devices. The dashed line indicates the value of $0.44 \mathrm{~mA} \mu \mathrm{m}^{-1}$ for $\mathrm{CVD}$-grown graphene from ref. 24. (g) SEM image of a typical FET device based on a monolayer GF with negative edge curvature. (h) $I_{D S}-V_{G S}$ curve measured at $V_{D S}=-0.01 \mathrm{~V}$ for a representative graphene FET device. (i) Hole mobility values for GFs with different edge curvatures. Each mobility value represents the result for one graphene FET device. The three regions separated by a dashed line in the graph show the results for different-shaped GFs, each of which is indicated by a schematic drawing inside the corresponding region. 
structure/property relationships. Furthermore, this truly $2 \mathrm{D}$ system is also amenable to simulation and theoretical studies, which should lead to a better understanding of the basic process of crystal growth under non-equilibrium conditions.

\section{CONFLICT OF INTEREST}

The authors declare no conflict of interest.

\section{ACKNOWLEDGEMENTS}

We thank Professor SX Du, Mr G Li and Professor HJ Gao for their valuable discussions and comments. This work was supported by the National Basic Research Program of China (2011CB932700, 2011CB808403, 2011CB932303, 2009CB623603), the National Natural Science Foundation of China (60911130231, 61171054, 21273243, 20973184, 20825208), and the Chinese Academy of Sciences.

1 Libbrecht, K. G. The physics of snow crystals. Rep. Prog. Phys 68, 855-895 (2005).

2 Yokoyama, E. \& Kuroda, T. Pattern formation in growth of snow crystals occurring in the surface kinetic process and the diffusion process. Phys. Rev. A 41, 2038-2049 (1990).

3 Saito, Y. Statistical Physics of Crystal Growth 66-123 (World Scientific, Singapore, 1996).

4 Roder, H., Hahn, E., Brune, H., Bucher, J. \& Kern, K. Building one-and twodimensional nanostructures by diffusion-controlled aggregation at surfaces. Nature 366, 141-143 (1993).

5 Zhang, Z. Y. \& Lagally, M. G. Atomistic processes in the early stages of thin-film growth. Science 276, 377-383 (1997).

6 Liberato, M., Delia, J. M., Andreas, M., Erik, C. S. \& Alivisatos, A. P. Controlled growth of tetrapod-branched inorganic nanocrystals. Nat. Mater. 2, 382-385 (2003).

7 Yin, Y. D. \& Alivisatos, A. P. Colloidal nanocrystal synthesis and the organic-inorganic interface. Nature 437, 664-670 (2005).

8 Li, C., Li, G., Shen, C. M., Hui, C., Tian, J. F., Du, S. X., Zhang, Z. Y. \& Gao, H. J. Atomic-scale tuning of self-assembled $\mathrm{ZnO}$ microscopic patterns: from dendritic fractals to compact island. Nanoscale 2, 2557-2560 (2010).

9 Novoselov, K. S., Geim, A. K., Morozov, S. V., Jiang, D., Zhang, Y., Dubonos, S. V., Grigorieva, I. V. \& Firsov, A. A. Electric field effect in atomically thin carbon films. Science 306, 666-669 (2004).

10 Geim, A. K. \& Novoselov, K. S. The rise of graphene. Nat. Mater. 6, 183-191 (2007). 11 Geim, A. K. Graphene Status and prospects. Science 324, 1530-1534 (2009).

12 Huang, P. Y., Ruiz-Vargas, C. S., Zande, A. M., Whitney, W. S., Levendorf, M. P., Kevek, J. W., Garg, S., Alden, J. S., Hustedt, C. J., Zhu, Y., Park, J., McEuen, P. L. \& Muller, D.
A. Grains and grain boundaries in single-layer graphene atomic patchwork quilts. Nature 469, 389-392 (2011).

13 Li, X. S., Cai, W. W., An, J. H., Kim, S., Nah, J Yang, D. X, Piner, R. Velamakanni, A. Jung, I., Tutuc, E., Banerjee, S. K., Colombo, L. G. \& Ruoff, R. S. Large-area synthesis of high-quality and uniform graphene films on copper foils. Science 324, 1312-1314 (2009).

14 Li, X. S., Cai, W. W., Colombo, L. G. \& Ruoff, R. S. Evolution of graphene growth on Ni and Cu by carbon isotope labelling. Nano Lett. 9, 4268-4272 (2009).

15 Yu, Q. K., Jauregui, L. A., Wu, W., Colby, R., Tian, J. F., Su, Z. H., Cao, H. L., Liu, Z. H., Pandey, D., Wei, D. G., Chung, T. F., Peng, P., Guisinger, N. P., Stach, E. A., Bao, J. M., Pei, S. S. \& Chen, Y. P. Control and characterization of individual grains and grain boundaries in graphene grown by chemical vapour deposition. Nat. Mater. 10, 443-449 (2011).

16 Robertson, A. W. \& Warner, J. H. Hexagonal single crystal domains of few-layer graphene on copper foils. Nano Lett. 11, 1182-1189 (2011).

17 Wu, B., Geng, D. C., Guo, Y. L., Huang, L. P., Xue, Y. Z., Zheng, J., Chen, J. Y., Yu, G., Liu, Y. Q., Jiang, L. \& Hu, W. P. Equiangular hexagon-shape-controlled synthesis of graphene on copper surface. Adv. Mater. 23, 3522-3525 (2011).

18 Wu, W., Jauregui, L. A., Su, Z. H., Liu, Z. H., Bao, J. M., Chen, Y. P. \& Yu, Q. K. Growth of single crystal graphene arrays by locally controlling nucleation on polycrystalline $\mathrm{Cu}$ using chemical vapor deposition. Adv. Mater. 23, 4898-4903 (2011).

19 Vlassiouk, I., Regmi, M., Fulvio, P., Dai, S., Datskos, P., Eres, G. \& Smirnov, S. Role of hydrogen in chemical vapor deposition growth of large single-crystal graphene. ACS Nano 5, 6069-6076 (2011).

20 Li, X. S., Magnuson, C. W., Venugopal, A., Tromp, R. M., Hannon, J. B., Vogel, E. M., Colombo, L. G. \& Ruoff, R. S. Large-area graphene single crystals grown by lowpressure chemical vapor deposition of methane on copper. J. Am. Chem. Soc. 133 , 2816-2819 (2011).

21 Li, X. S., Magnuson, C. W., Venugopal, A., An, J. H., Suk, J. W., Han, B. Y., Borysiak, M., Cai, W. W., Velamakanni, A., Zhu, Y. W., Fu, L. F., Vogel, E. M., Colombo, L. G. \& Ruoff, R. S. Graphene films with large domain size by a two-step chemical vapor deposition process. Nano Lett. 10, 4328-4334 (2010).

22 Wofford, J. M., Nie, S., McCarty, K. F., Bartelt, N. C. \& Dubon, O. D. Graphene islands on $\mathrm{Cu}$ foils: the interplay between shape, orientation, defects. Nano Lett. 10, 4890-4896 (2010).

23 Geng, D. C., Wu, B., Guo, Y. L., Huang, L. P., Xue, Y. Z., Chen, J. Y., Yu, G., Jiang, L., Hu, W. P. \& Liu, Y. Q. Uniform hexagonal graphene flakes and films grown on liquid copper surface. Proc. Natl Acad. Sci. USA 109, 7992-7996 (2012).

24 Bai, J. W., Liao, L., Zhou, H. L., Cheng, R., Liu, L. X., Huang, Y. \& Duan, X. F. Top-gated chemical vapor deposition grown graphene transistors with current saturation. Nano Lett. 11, 2555-2559 (2011).

(c) (1) () $\Theta$ This work is licensed under a Creative Commons Attribution-NonCommercial-NoDerivs 3.0 Unported License. To view a copy of this license, visit http://creative commons.org/licenses/by-nc-nd/3.0/

Supplementary Information accompanies the paper on the NPG Asia Materials website (http://www.nature.com/am) 